\title{
Iridabenzenes from Iridacyclopentadienes: Unusual C-C Bond Formation between Unsaturated Hydrocarbyl Ligands
}

Chong Shik Chin,* Hyungeui Lee and Min-Sik Eum

Chemistry Department, Sogang University, Seoul 121-742, Korea, Fax: +82-2-701-0967; Tel: +82-2-705-8448;

E-mail: cschin@sogang.ac.kr

\section{SUPPORTING INFORMATION}

Table of Contents

${ }^{1} \mathrm{H}$ NMR spectrum of 4 at $500 \mathrm{MHz}$ in $\mathrm{CDCl}_{3} \ldots \ldots \ldots \ldots \ldots \ldots \ldots \ldots \ldots \ldots \ldots$

${ }^{13} \mathrm{C}$ NMR spectrum of 4 at $126 \mathrm{MHz}$ in $\mathrm{CDCl}_{3} \ldots \ldots \ldots \ldots \ldots \ldots \ldots \ldots \ldots$

${ }^{1} \mathrm{H}$ NMR spectrum of $5 \mathbf{a}$ at $500 \mathrm{MHz}$ in $\mathrm{CDCl}_{3} \ldots \ldots \ldots \ldots \ldots \ldots \ldots \ldots \ldots$

${ }^{1} \mathrm{H}$ NMR spectrum of $\mathbf{5 a - d ( C 4 )}$ at $500 \mathrm{MHz}$ in $\mathrm{CDCl}_{3} \ldots \ldots \ldots \ldots \ldots \ldots \ldots$

${ }^{13} \mathrm{C}$ NMR spectrum of $\mathbf{5 a}$ at $126 \mathrm{MHz}$ in $\mathrm{CDCl}_{3} \ldots \ldots \ldots \ldots \ldots \ldots \ldots \ldots \ldots$

${ }^{1} \mathrm{H}$ NMR spectrum of $\mathbf{6 a}$ at $500 \mathrm{MHz}$ in $\mathrm{CDCl}_{3} \ldots \ldots \ldots \ldots \ldots \ldots \ldots \ldots \ldots \ldots \ldots \ldots$

${ }^{1} \mathrm{H}$ NMR spectrum of $\mathbf{6 a}-\mathbf{d}(\mathbf{C 6})$ at $300 \mathrm{MHz}$ in $\mathrm{CDCl}_{3} \ldots \ldots \ldots \ldots \ldots \ldots \ldots$

${ }^{1} \mathrm{H}$ NMR spectrum of $\mathbf{6 a}-\mathbf{d}\left(\mathbf{C 4 , C 6 )}\right.$ at $300 \mathrm{MHz}$ in $\mathrm{CDCl}_{3} \ldots \ldots \ldots \ldots$ 9S

${ }^{13} \mathrm{C}$ NMR spectrum of $\mathbf{6 a}$ at $126 \mathrm{MHz}$ in $\mathrm{CDCl}_{3} \ldots \ldots \ldots \ldots \ldots \ldots \ldots \ldots \ldots \ldots \ldots$

${ }^{1} \mathrm{H}$ NMR spectrum of $\operatorname{Ir}(-\mathrm{C} \equiv \mathrm{C}-\mathrm{Ph})_{2}\left(\eta^{2}-\mathrm{O}_{2} \mathrm{CCH}_{3}\right)\left(\mathrm{PPh}_{3}\right)_{2}$ at $500 \mathrm{MHz}$ in $\mathrm{CDCl}_{3} \ldots \ldots \ldots$ 11S

${ }^{13} \mathrm{C}$ NMR spectrum of $\operatorname{Ir}(-\mathrm{C} \equiv \mathrm{CPh})_{2}\left(\eta^{2}-\mathrm{O}_{2} \mathrm{CCH}_{3}\right)\left(\mathrm{PPh}_{3}\right)_{2}$ at $126 \mathrm{MHz}$ in $\mathrm{CDCl}_{3} \ldots \ldots \ldots . \quad 12 \mathrm{~S}$ 


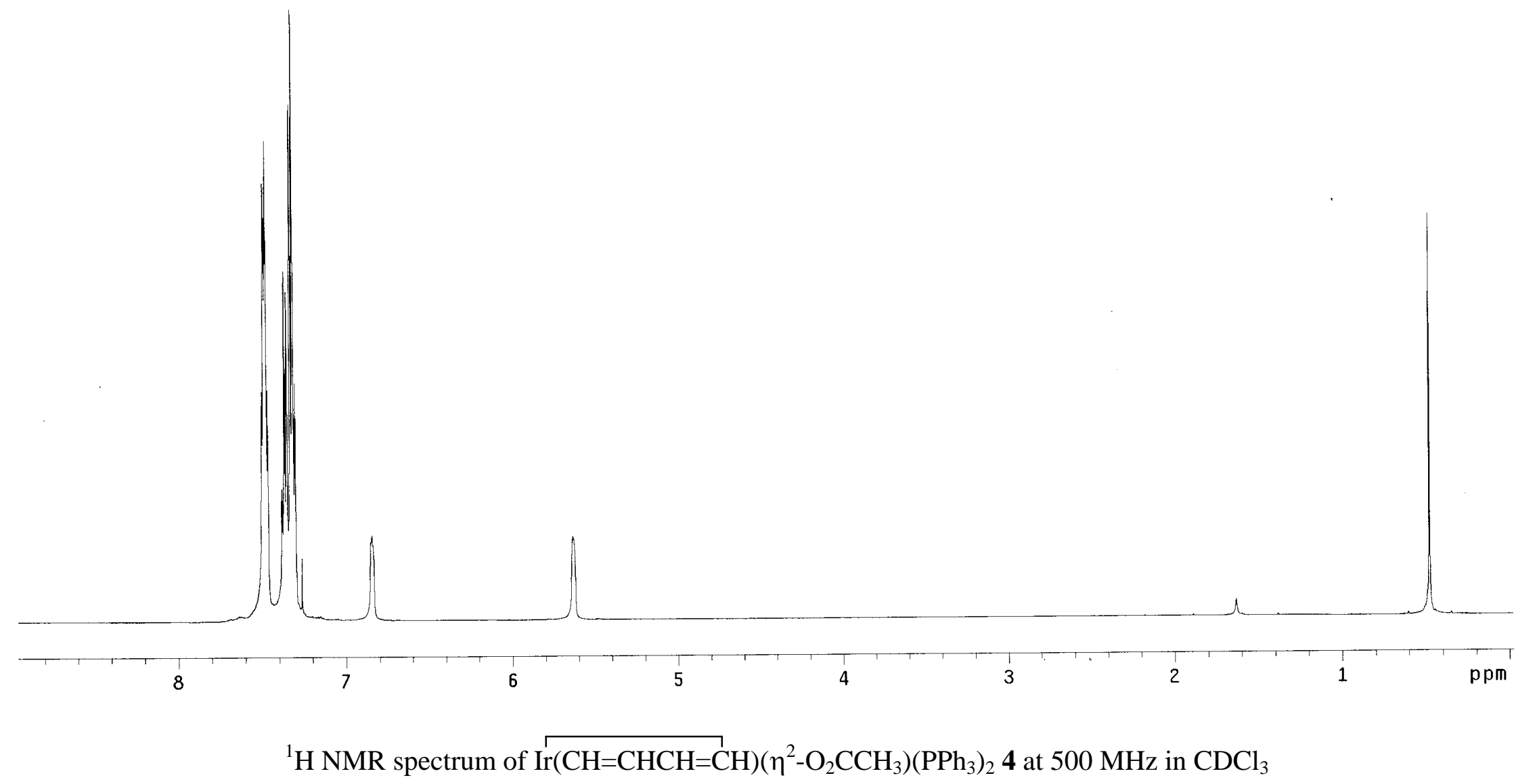




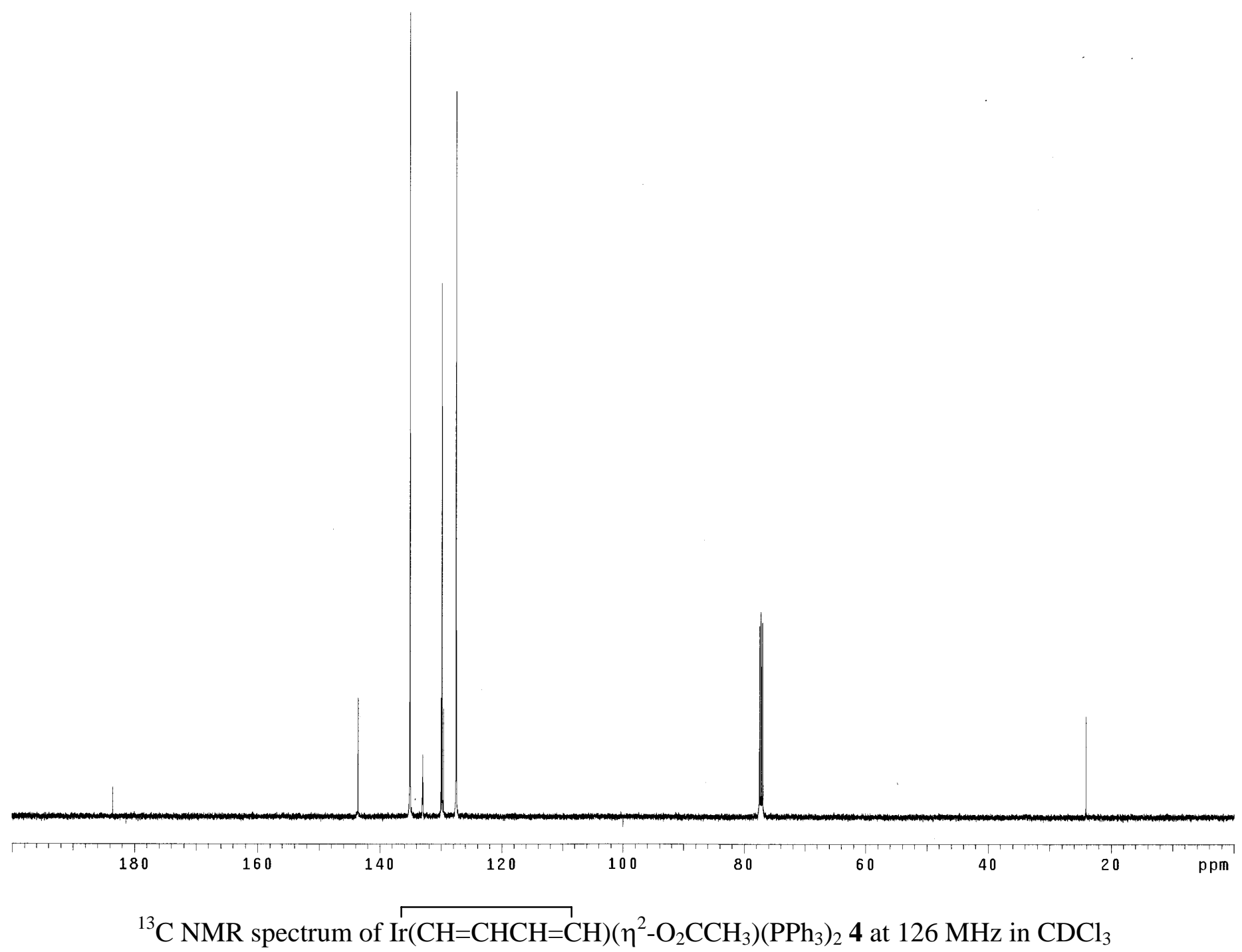




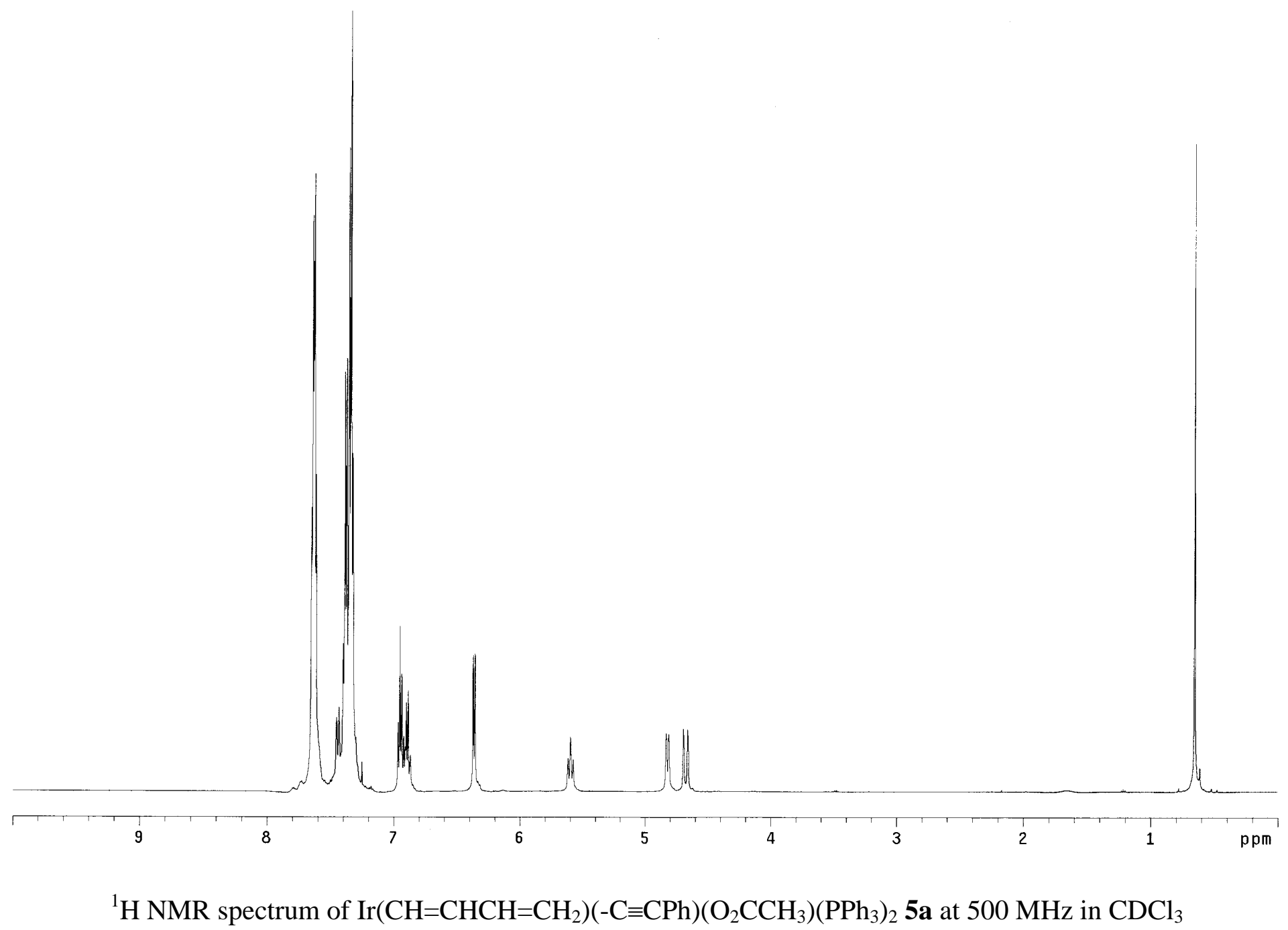




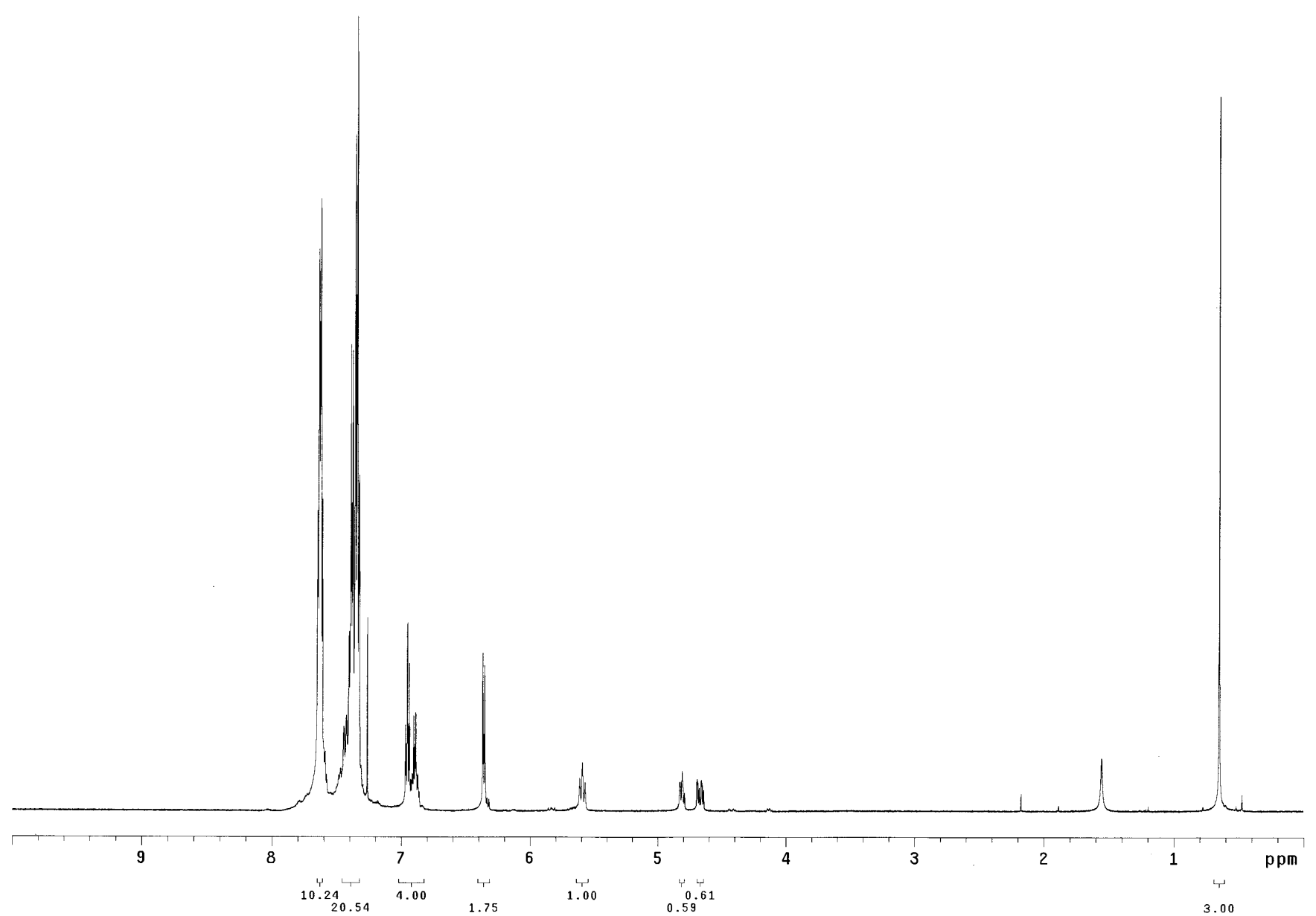

${ }^{1} \mathrm{H}$ NMR spectrum of $\operatorname{Ir}(\mathrm{CH}=\mathrm{CHCH}=\mathrm{CHD})(-\mathrm{C} \equiv \mathrm{CPh})\left(\eta^{2}-\mathrm{O}_{2} \mathrm{CCH}_{3}\right)\left(\mathrm{PPh}_{3}\right)_{2} \mathbf{5 a - d}(\mathbf{C} 4)$ at $500 \mathrm{MHz}$ in $\mathrm{CDCl}_{3}$ 


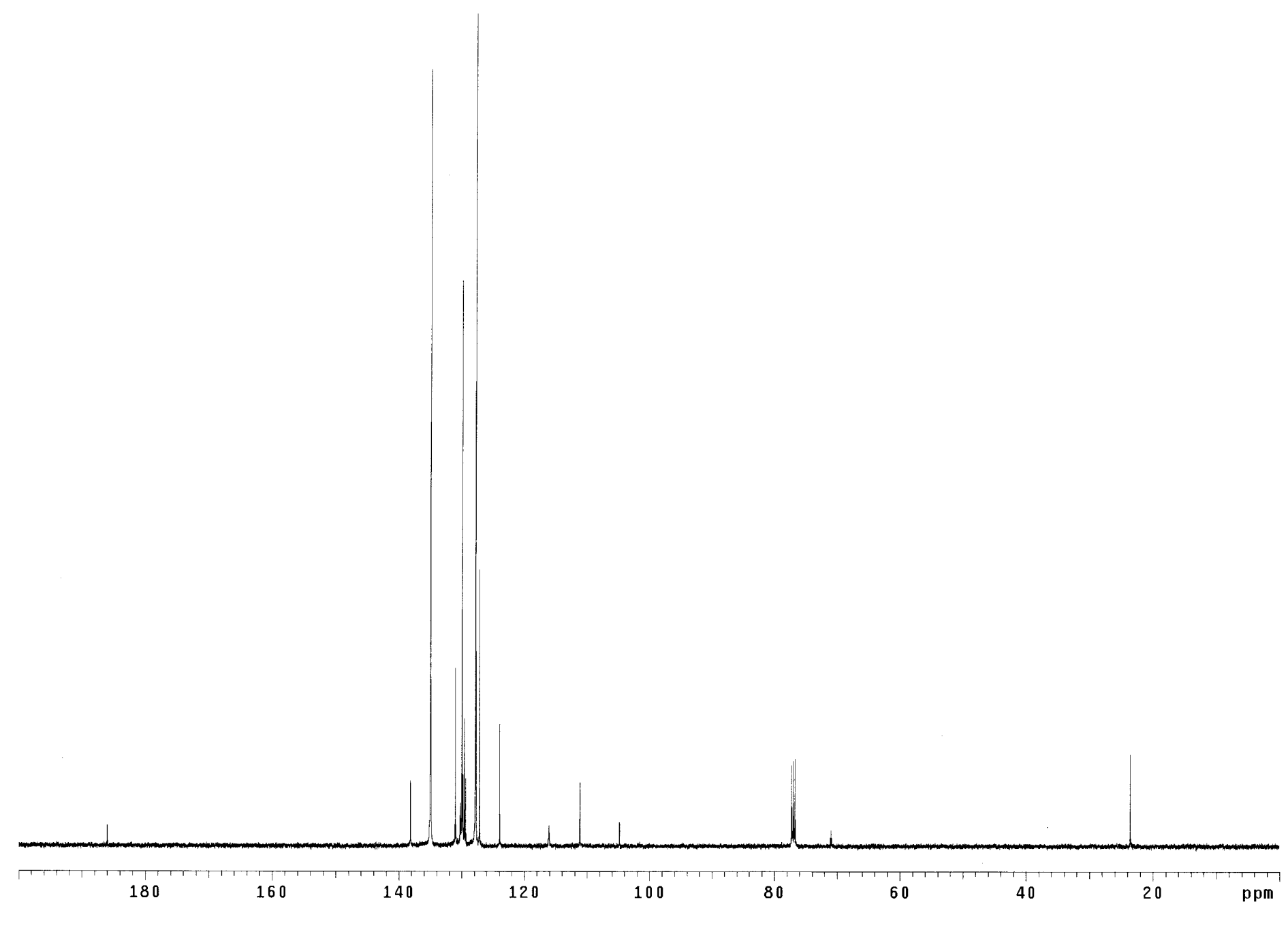

${ }^{13} \mathrm{C}$ NMR spectrum of $\operatorname{Ir}\left(\mathrm{CH}=\mathrm{CHCH}=\mathrm{CH}_{2}\right)(-\mathrm{C} \equiv \mathrm{CPh})\left(\eta^{2}-\mathrm{O}_{2} \mathrm{CCH}_{3}\right)\left(\mathrm{PPh}_{3}\right)_{2} \mathbf{5 a}$ at $125.7 \mathrm{MHz}$ in $\mathrm{CDCl}_{3}$ 


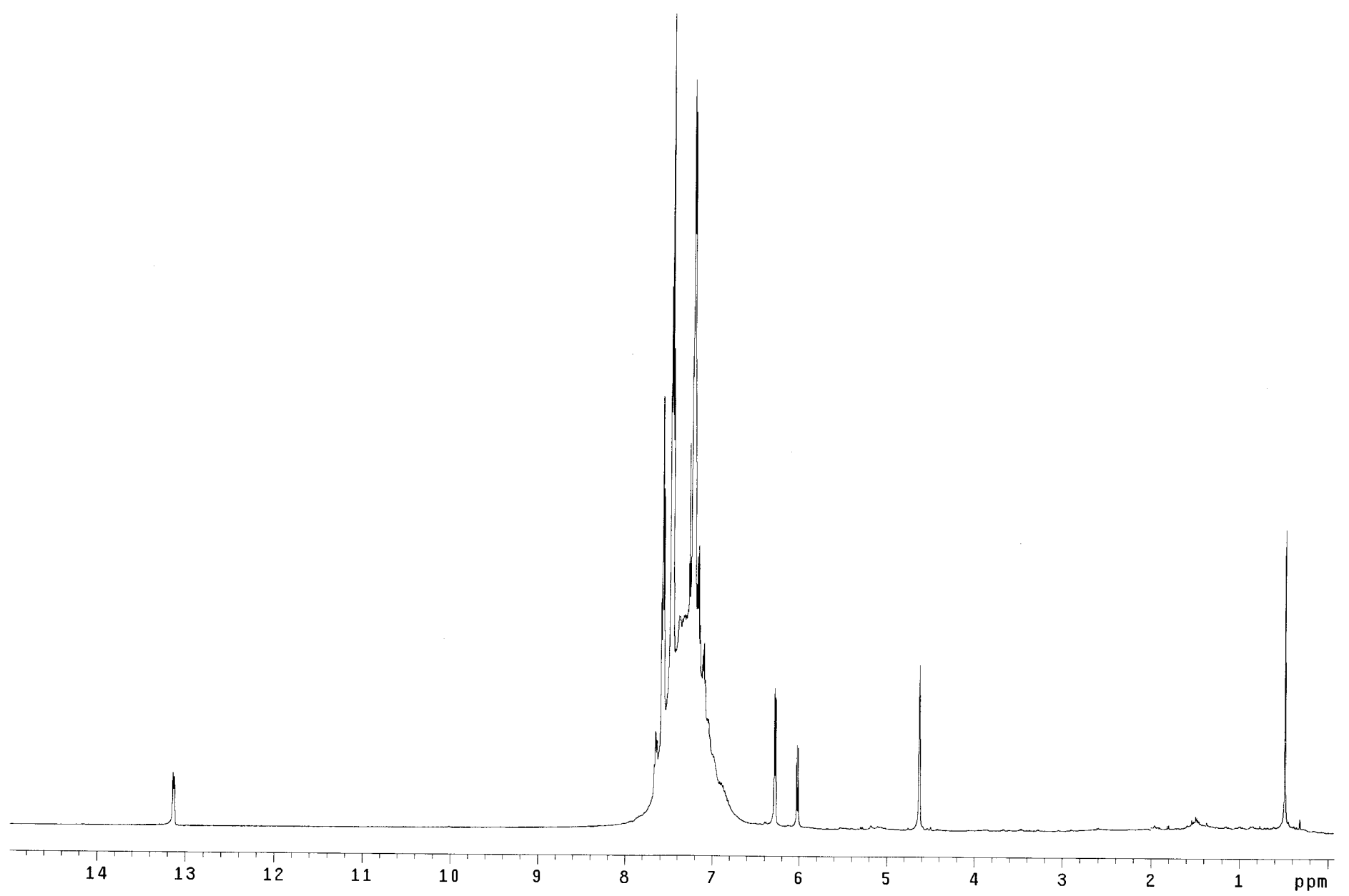

${ }^{1} \mathrm{H}$ NMR spectrum of $\left[\mathrm{Ir}=\mathrm{CH}-\mathrm{CH}=\mathrm{CH}-\mathrm{CH}=\mathrm{C}\left(\mathrm{CH}_{2} \mathrm{Ph}\right)\left(\eta^{2}-\mathrm{O}_{2} \mathrm{CCH}_{3}\right)\left(\mathrm{PPh}_{3}\right)_{2}\right] \mathrm{OTf} \mathbf{6 a}$ at $500 \mathrm{MHz}$ in $\mathrm{CDCl}_{3}$ 


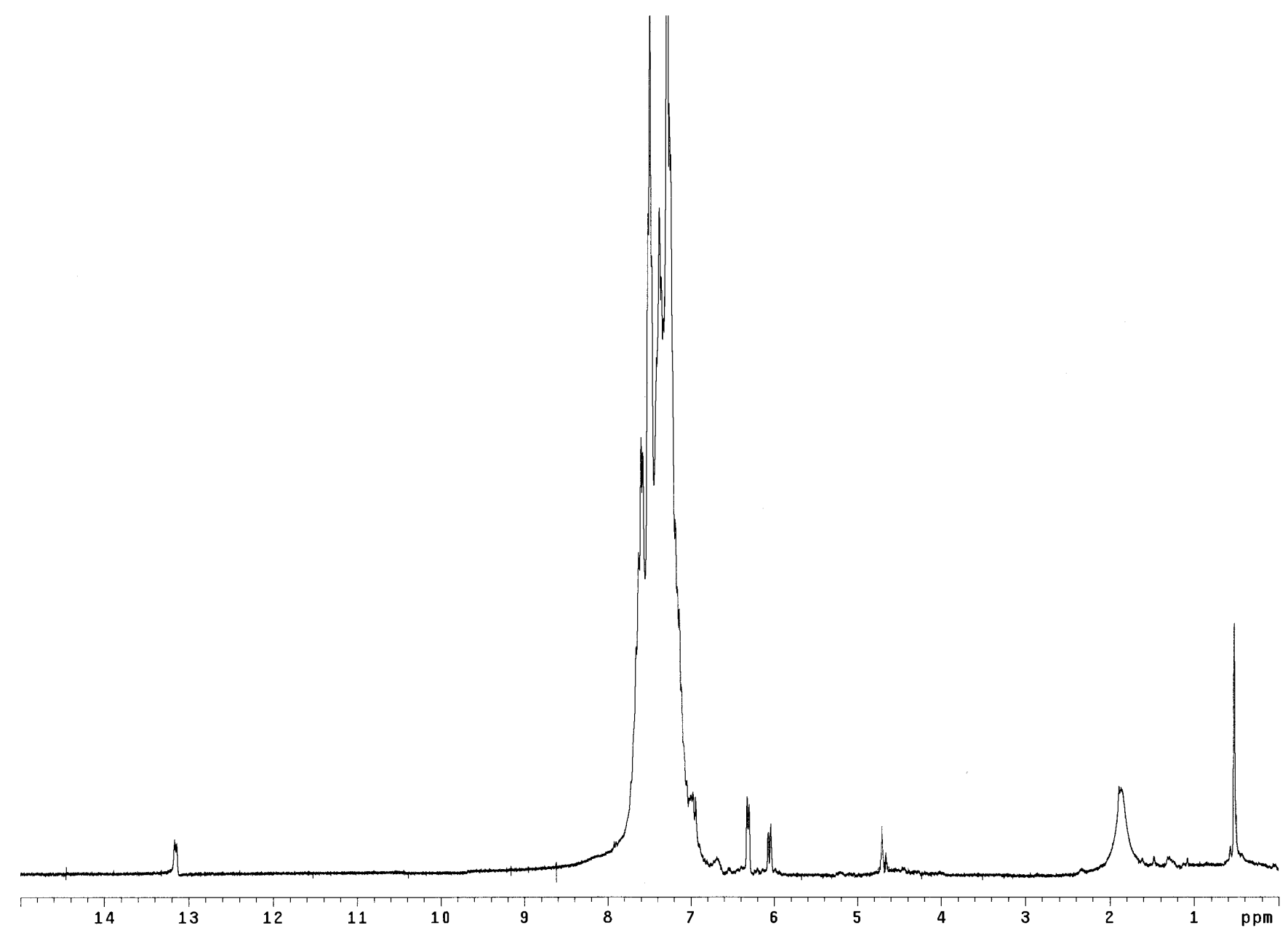

${ }^{1} \mathrm{H}$ NMR spectrum of $\left[\mathrm{Ir}=\mathrm{CH}-\mathrm{CH}=\mathrm{CH}-\mathrm{CH}=\mathrm{C}(\mathrm{CHDPh})\left(\eta^{2}-\mathrm{O}_{2} \mathrm{CCH}_{3}\right)\left(\mathrm{PPh}_{3}\right)_{2}\right] \mathrm{OTf} \mathbf{6 a -}-\boldsymbol{d}(\mathbf{C 6})$ at $300 \mathrm{MHz}$ in $\mathrm{CDCl}_{3}$ 


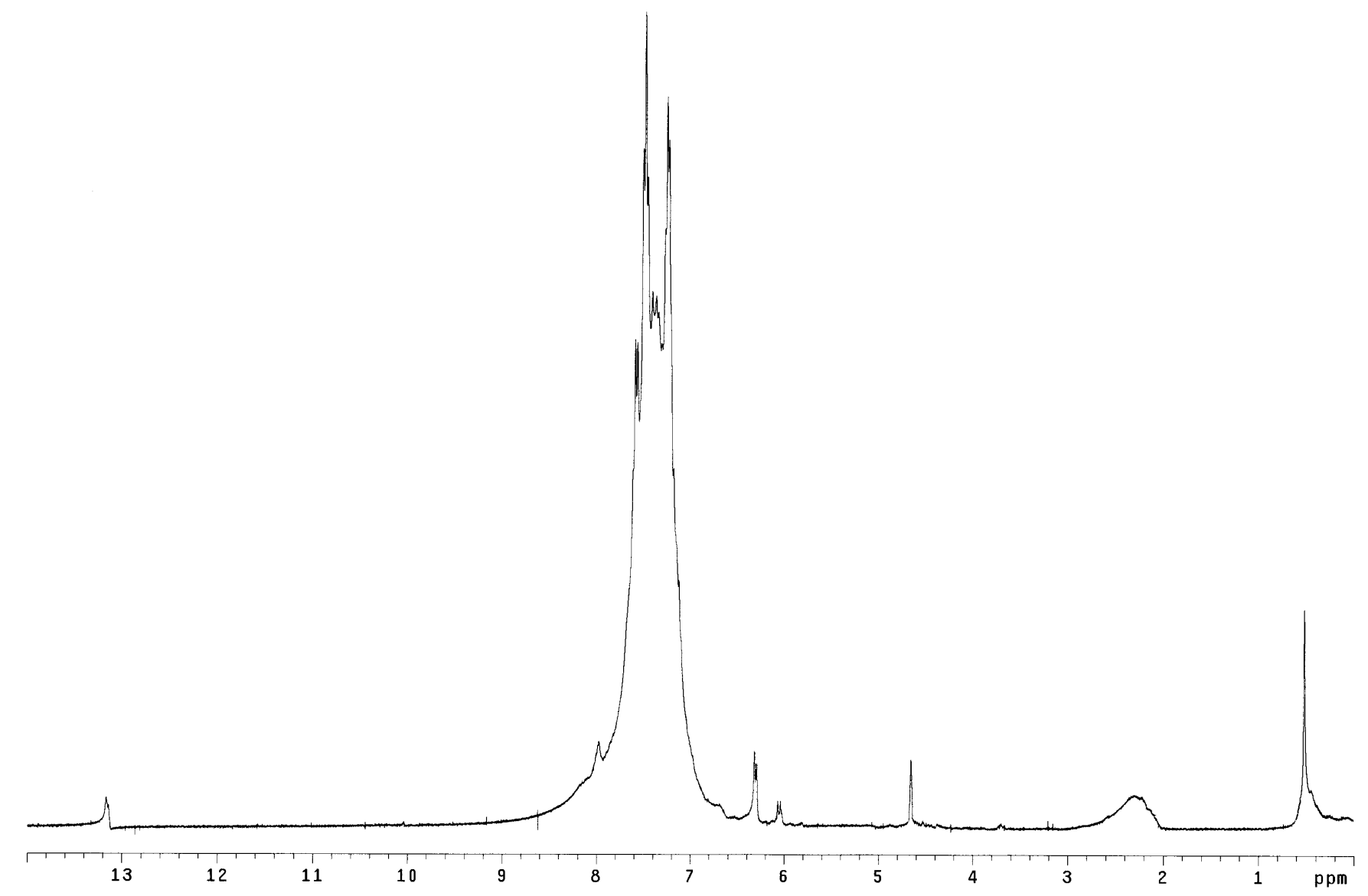

${ }^{1} \mathrm{H}$ NMR spectrum of $\left[\mathrm{Ir}=\mathrm{CH}-\mathrm{CH}=\mathrm{CH}-\mathrm{CD}_{\mathrm{y}} \mathrm{H}_{1-\mathrm{y}}=\mathrm{C}\left(\mathrm{CH}_{2-\mathrm{x}} \mathrm{D}_{\mathrm{x}} \mathrm{Ph}\right)\left(\eta^{2}-\mathrm{O}_{2} \mathrm{CCH}_{3}\right)\left(\mathrm{PPh}_{3}\right)_{2}\right] \mathrm{OTf} \mathbf{6 a - d}(\mathbf{C} 4, \mathbf{C 6})(0.5<\mathrm{x}+\mathrm{y}<1)$ at $300 \mathrm{MHz}$ in $\mathrm{CDCl}_{3}$ 


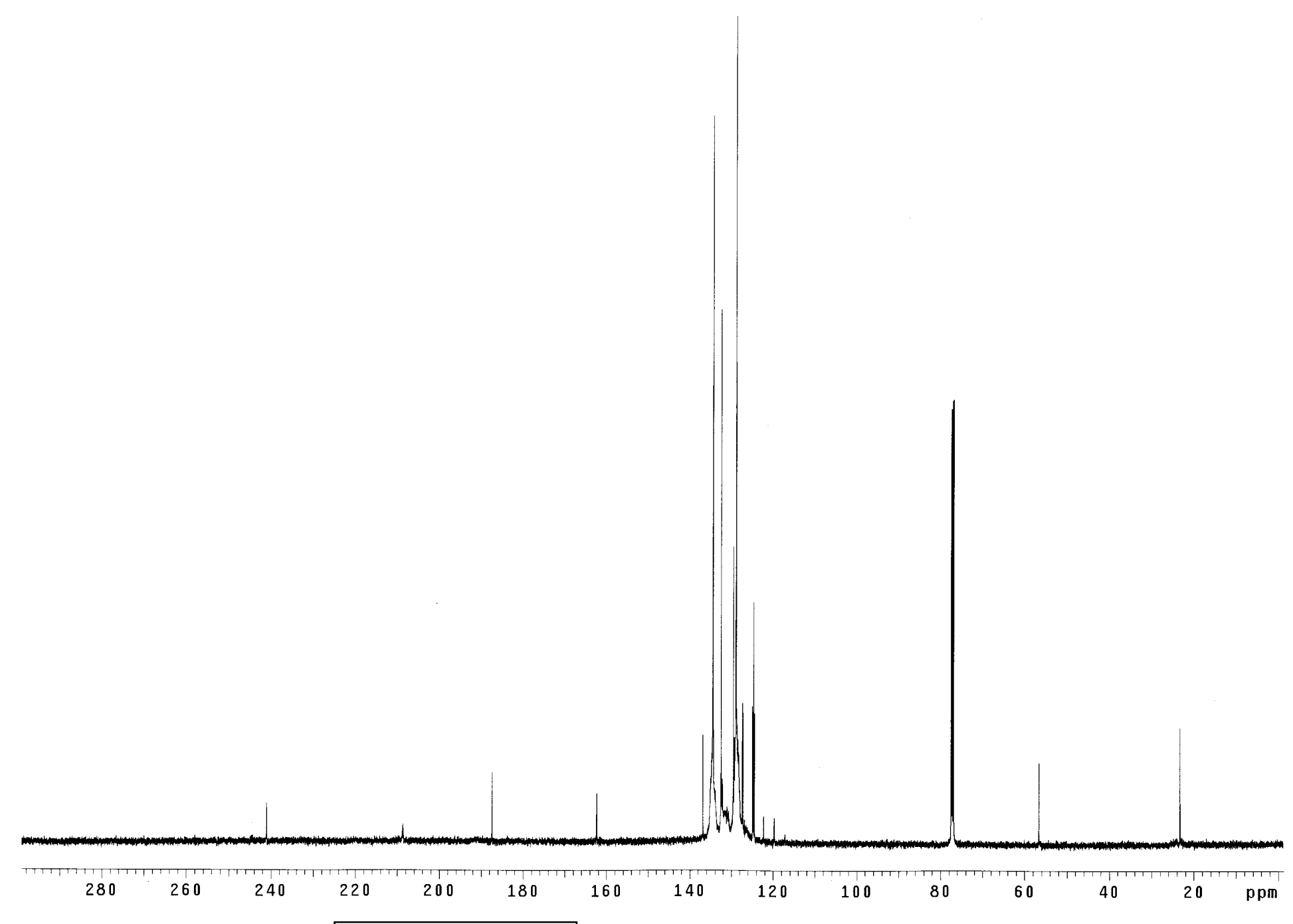

${ }^{13} \mathrm{C}$ NMR spectrum of $\left[\mathrm{Ir}=\mathrm{CH}-\mathrm{CH}=\mathrm{CH}-\mathrm{CH}=\mathrm{C}\left(\mathrm{CH}_{2} \mathrm{Ph}\right)\left(\eta^{2}-\mathrm{O}_{2} \mathrm{CCH}_{3}\right)\left(\mathrm{PPh}_{3}\right)_{2}\right] \mathrm{OTf}$ 6a at $126 \mathrm{MHz}$ in $\mathrm{CDCl}_{3}$ 


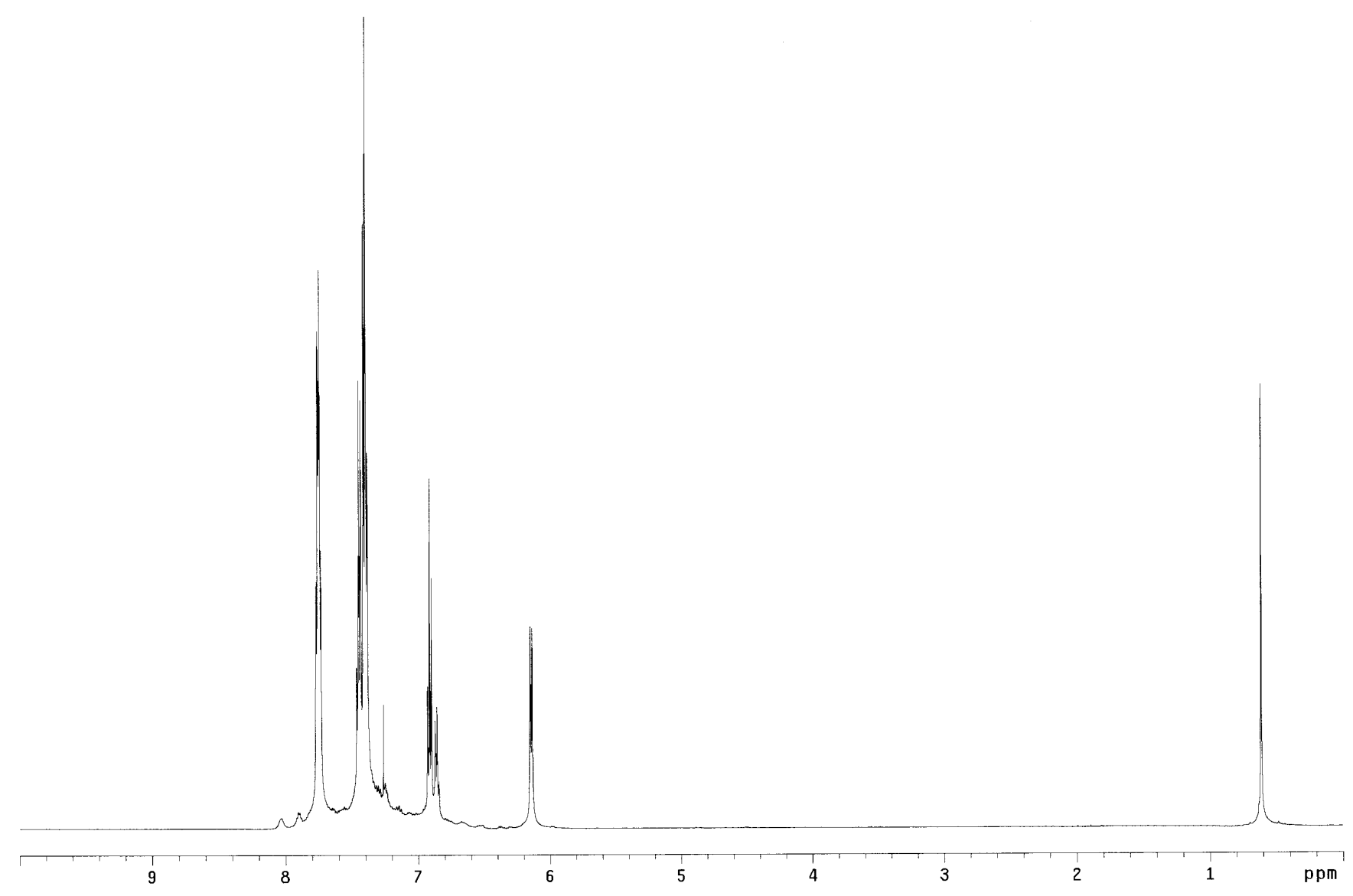

${ }^{1} \mathrm{H}$ NMR spectrum of $\operatorname{Ir}(-\mathrm{C} \equiv \mathrm{C}-\mathrm{Ph})_{2}\left(\eta^{2}-\mathrm{O}_{2} \mathrm{CCH}_{3}\right)\left(\mathrm{PPh}_{3}\right)_{2}$ at $500 \mathrm{MHz}$ in $\mathrm{CDCl}_{3}$ 


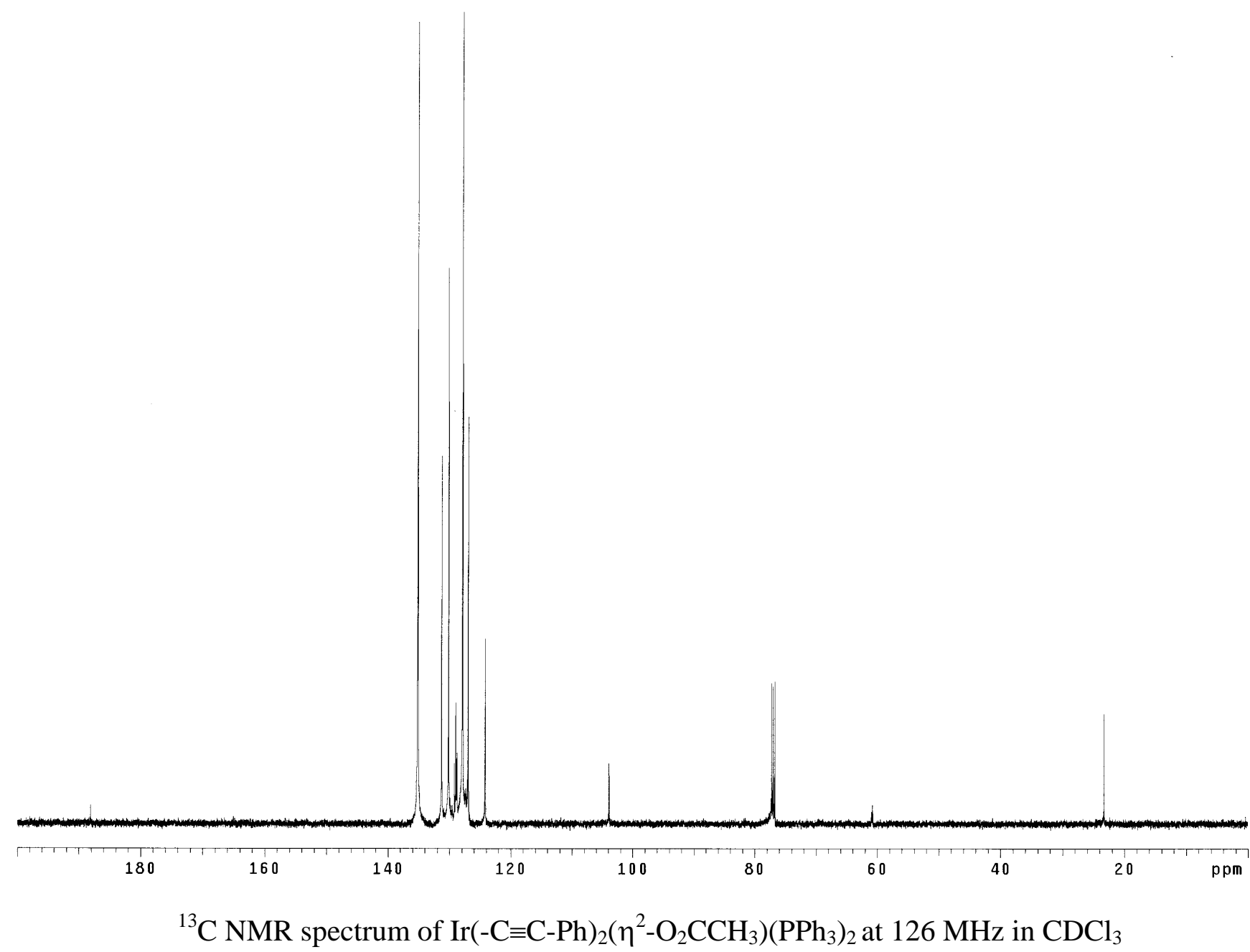

